Mots. Les langages du politique

$101 \mid 2013$

Le discours politique portugais

\title{
Un discours politique européen
}

Paul Bacot, Maria-Aldina Marques et Michèle Monte

\section{OpenEdition}

Journals

Édition électronique

URL : https://journals.openedition.org/mots/21093

DOI : $10.4000 /$ mots. 21093

ISSN : 1960-6001

\section{Éditeur}

ENS Éditions

\section{Édition imprimée}

Date de publication : 22 avril 2013

Pagination : 5-11

ISBN : 978-2-84788-393-0

ISSN : 0243-6450

\section{Référence électronique}

Paul Bacot, Maria-Aldina Marques et Michèle Monte, « Un discours politique européen », Mots. Les langages du politique [En ligne], 101 | 2013, mis en ligne le 22 avril 2015, consulté le 22 avril 2022. URL : http://journals.openedition.org/mots/21093; DOI : https://doi.org/10.4000/mots.21093 


\section{Un discours politique européen}

Pendant quelques mois, en 1974, le Portugal fut au centre du débat politique en France. C'est peu dire que les Français s'intéressèrent alors à la politique portugaise. Un système complexe d'homologies permettait à chaque courant hexagonal de se retrouver dans les discours d'un leader lusitanien : Otelo de Carvalho, le général Spínola, Álvaro Cunhal, Mário Soares...

Seule la présidence de Salvatore Allende, quelques années auparavant, avait suscité ce phénomène de quasi-fusion de la vie politique française avec une vie politique étrangère. Si une commune latinité a pu faciliter cette intercompréhension, d'ailleurs nécessairement limitée, tant les différences entre les pays étaient alors considérables, on peut s'interroger sur les raisons d'un intérêt aussi vif. La présence de nombreux immigrés portugais en France, notamment de responsables politiques exilés pendant la dictature, l'existence dans les deux pays d'un important Parti communiste qui pouvait faire craindre à certains une bascule vers le camp soviétique, les tentatives d'organisation autogestionnaire de la production qui ont vu le jour dans les années 1974-1975 ont sans doute joué un rôle. Enfin, et peut-être surtout, le souvenir encore tout proche de Mai 1968 avec ses débats sur les différentes formes de révolution, et l'existence en France d'un programme commun de gouvernement PS-PC, sont alors des circonstances déterminantes.

Mais il y a longtemps que le Portugal issu de la Révolution des Fillets ne suscite plus la passion chez le citoyen français. On pourrait même dire que c'est une assez grande indifférence, doublée d'une bonne dose d'ignorance, qui caractérise le regard porté en France sur la vie politique portugaise. Même les effets de la crise sur le pays n'ont pas suffi à attirer l'attention des médias. Le sort de la Grèce, il est vrai encore plus durement touchée, a davantage retenu leur attention et, pour autant qu'on puisse en juger, celle de l'homme de la rue.

La chute de la dictature salazariste et la décolonisation, puis la fin des remous postrévolutionnaires, et enfin l'entrée dans la Communauté économique

Université de Lyon, IEP, UMR 5206 Triangle

paul.bacot@sciencespo-lyon.fr

Universidade do Minho, Portugal

mamarques@ilch.uminho.pt

Université de Toulon, Babel EA 2649

michele.monte@univ-tln.fr 
européenne en 1986, puis dans la zone euro en 1999, ont en quelque sorte banalisé le Portugal, européen parmi les autres, même si, comme les autres, il a ses spécificités, y compris sur le plan constitutionnel (Garnier, 2001). Voici une République dont le président est élu au suffrage universel direct, mais qui ne préside pas le conseil des ministres. Voici un régime parlementaire, mais unicaméral. Voici un système partisan bipolaire avec, d'un côté, un Parti socialiste, mais de l'autre - à droite - un parti qui s'intitule «social-démocrate», parfois allié à un parti plus à droite nommé «Centre démocratique et social ». Et sur la gauche du Parti socialiste, à la fois un Parti communiste et un «Bloc de gauche» qui n'ont jamais conclu d'alliance, ni entre eux, ni avec le Parti socialiste. Voici, enfin, un pays qui, après avoir perdu le Brésil au $19^{e}$ siècle par une scission de sa propre famille royale, et tourné la page de la décolonisation à la faveur d'une révolution qui a conduit à assimiler colonialisme et dictature, évoque avec fierté son passé d'expansion maritime : il y puise des raisons d'espérer un nouveau dynamisme, alors que l'émigration reprend et que la mondialisation fragilise son récent développement économique.

On le voit, la politique lusitanienne, et notamment ses manifestations discursives, méritent à coup sûr qu'on y prête intérêt. Comment se construit verbalement cette vie politique à la fois banale et spécifique? L'existence dans le pays même d'une communauté scientifique travaillant sur ce sujet permet à ce dossier de privilégier un regard de l'intérieur.

L'intérêt scientifique porté actuellement à l'analyse du discours, au Portugal, se manifeste dans un cadre qui, d'une manière générale, ne diffère pas du cadre international. Toutefois, l'analyse du discours, et du discours politique en particulier, accompagne l'histoire de la démocratie portugaise. C'est avec la Révolution d'avril 1974 que le discours politique a gagné une centralité sociale indéniable, suscitant l'intérêt de chercheurs des différents domaines des sciences humaines et sociales et ouvrant la voie à un processus d'affirmation progressive de l'analyse du discours politique en tant que domaine de recherche pluridisciplinaire.

Les travaux de sociologie politique qui se sont développés récemment se sont très logiquement intéressés aux scrutins (Freire, Magalhães, 2002 ; Freire, Lobo, Magalhães, 2007), à l'organisation des partis, à leur implantation et à leur trajectoire (Belchior, 2010 ; Jalali, 2007; Lisi, 2011), avec fréquemment une dimension comparative (Lopes, 2004; Freire, 2006), mais sans porter pour l'instant une grande attention aux pratiques discursives, hormis Filipe (2002).

L'analyse du discours politique en tant que tel s'est développée davantage dans le champ des sciences du langage et des sciences de la communication. Le discours politique parlementaire et le discours politique de campagnes électorales successives sont probablement les domaines les plus étudiés, bien que l'intérêt scientifique pour toutes les manifestations politiques discur- 
sives soit dûment documenté. La bibliographie sur le discours politique portugais témoigne de la diversité de ces perspectives et de la systématicité de la recherche. Parmi les références non exhaustives que nous proposons à la fin de cette introduction, nous soulignerons à titre d'exemple les thèses de doctorat sur le discours politique, dont la première (Marques, 1988) avait pour objet les discours parlementaires, de l'Assemblée nationale à la fin de la dictature (19721974) jusqu'à la fin de l'Assemblée constituante (1974-1975) : dans une perspective sociolinguistique, y sont étudiées, en particulier, les formes d'adresse. En 2000, une autre thèse de doctorat (Marques, 2000) aborde la construction énonciative des débats parlementaires. L'auteure consacre ensuite plusieurs travaux à l'image de soi et de l'autre dans différents genres de discours politiques (Marques, 2007, 2010 a et b). D'autres travaux sur les émotions et les discours politiques (Pinto, 2005 et Marques, 2012) ou bien sur les caractéristiques des rapports entre discours politique et discours médiatique (Duarte, 2011 et Ramos, 2011) témoignent de l'intérêt suscité par ce genre de discours dans le domaine des sciences du langage. Dans les travaux de communication politique, on mentionnera Sena (2002) et Salgado (2010), qui traitent respectivement des débats télévisés et de la construction de l'image des candidats. Du côté des sciences sociales, Espírito Santo (2006 et 2007) s'intéresse aux discours présidentiels d'investiture, selon une méthodologie d'analyse de contenu d'inspiration américaine.

Le dossier de ce numéro comprend quatre articles qui portent tous sur la période la plus récente de la vie politique portugaise mais qui s'intéressent à des productions sémio-linguistiques différentes : discours d'investiture des Premiers ministres de 2002 à 2009, vœux présidentiels en 2008, 2009 et 2010, affiches électorales en 2002 et 2011, et émission de télévision pendant la campagne électorale pour les législatives de 2009. Les trois premiers articles considèrent le discours politique en lui-même en tant qu'entreprise de légitimation à destination des citoyens alors que le quatrième l'envisage dans ses rapports avec les médias.

L'article de Michèle Monte intitulé « Les discours d'investiture des Premiers ministres portugais de 2002 à 2011. Comparaison gauche/droite» compare cinq discours, trois émanant de leaders du Parti social démocrate, de centre droit, et deux du leader du Parti socialiste dans la période 2005-2011. Ces discours sont certes prononcés devant le président de la République et les corps constitués et c'est à eux qu'ils sont adressés, mais leur destinataire indirect est sans nul doute l'ensemble du peuple portugais qui vient, par les élections législatives, de choisir le parti majoritaire à l'Assemblée et par là même le Premier ministre qui gouvernera le pays. Le discours d'investiture contribue à la construction de l'image du leader et à sa légitimation en proposant simultanément une certaine vision de la situation du pays et en présentant les 
orientations que le gouvernement compte suivre. L'article procède ainsi à une comparaison du lexique et de l'organisation des cinq discours afin de déterminer si les différences gauche/droite influent sur les schématisations discursives (Grize, 1990) ainsi construites. Il ressort de la comparaison que, malgré des conceptions du rôle de l'État différentes voire opposées, les représentations de la crise et des relations entre le gouvernement et le pays s'avèrent souvent très proches, ce qui donne à ces discours un air de famille peu propice à motiver les citoyens pour la chose politique.

L'article d'Andreea Teletin intitulé « Les vœux présidentiels au Portugal, en France et en Roumanie, et la crise internationale » et sous-titré "Les enjeux des formes d'adresse et des procédés d'atténuation/intensification» adopte, lui aussi, une perspective comparative, mais porte sur les discours de vœux, un genre discursif bien étudié en France. L'absence d'analyse lexicométrique ne permet pas vraiment de comparer la répartition des formes personnelles avec celle observée par Leblanc (2003), mais l'étude fait ressortir le poids du rituel, d'une part, celui du contexte socio-économique, d'autre part, tout en montrant aussi que certains choix sont liés à la singularité des ethos. Si la spécificité des discours roumains s'explique en grande partie par la volonté de rompre avec le rituel pesant du régime totalitaire, l'évocation des citoyens affligés par la crise, telle qu'elle apparaît par exemple chez Cavaco Silva, s'inscrit quant à elle dans une tradition d'empathie bien soulignée par Leblanc et Martinez (2005).

Rosalice Pinto nous propose, pour sa part, la comparaison de deux affiches électorales du Parti socialiste lors de la campagne pour les législatives de 2002 et 2011. L'exiguïté du corpus ne permet pas de donner aux lecteurs une vision plus large des tendances à l'œuvre au Portugal dans ce mode de communication politique ${ }^{1}$, mais l'auteure attire à juste titre notre attention sur l'utilisation des couleurs. La campagne de Ferro Rodrigues en 2002 est bâtie sur le contraste statique du vert et du rouge (les couleurs du drapeau portugais) alors que les affiches de José Sócrates en 2009, qui cherchent à donner une impression de mouvement, proposent une version plus dynamique de ce contraste en jouant sur le dégradé.

Dans son article «Politique, humour et campagne électorale. Les enjeux d'une politique-spectacle», Maria Aldina Marques analyse la participation des différents leaders des partis politiques à une émission humoristique télévisée. Elle étudie le type de relation que les invités construisent avec l'animateur et les contraintes qui pèsent sur eux pendant l'interaction : nécessité de réagir avec

1. On trouvera sur http://politikaeimagens.blogspot.pt un éventail assez large mais très hétéroclite d'affiches politiques des différents partis portugais. Si le rouge et vert est emblématique du PS, le bleu est la couleur privilégiée par le PC et la coalition CDU qu'il dirige, mais aussi par le CDS (parti de droite), alors que l'orange est la couleur du PSD depuis sa fondation. On observe cependant que les élections présidentielles ne mobilisent pas du tout la même répartition des couleurs que les autres élections, comme si l'identité des partis passait à l'arrière-plan au profit des valeurs incarnées par le candidat. 
humour aux blagues ou provocations de l'humoriste, juste dosage du sérieux et de la décontraction. S'ils sont prêts à courir le risque de mettre en danger leur image, c'est qu'ils escomptent de ce passage à la télévision un bénéfice important : celui de donner l'impression d'une proximité avec les électeurs. Mais ce moyen d'attirer les suffrages n'est pas sans danger, d'autant que l'interview des leaders politiques succède à une première partie d'émission deux fois plus longue qui porte sur les derniers épisodes de la campagne un regard satirique ou décalé dont le personnel politique fait les frais.

On constate à la lecture des quatre articles que les questions qui se posent aux analystes de la vie politique portugaise ne sont pas différentes de celles qui sont débattues ailleurs : affaiblissement des différences gauche/droite, euphémisation des problèmes dans un discours ritualisé, recours aux procédés publicitaires dans les campagnes électorales, poids des médias imposant leurs formes de communication aux politiques. Le parcours historique que nous avons esquissé dans cette introduction explique ces similitudes : en s'installant dans une alternance entre le PSD et le PS, et en entrant dans l'Union européenne dont il a adopté les choix économiques, le Portugal a fortement réduit sa singularité. Il a par ailleurs adopté des modes de communication politique largement répandus dans tous les pays occidentaux. Il existe certainement des spécificités portugaises que d'autres études auraient pu permettre de souligner, mais, pour mieux les appréhender, il faudrait développer les collaborations et les travaux comparatifs. En attirant l'attention sur ce pays, ce dossier pourra peut-être susciter de telles recherches. Nous espérons par ailleurs qu'il incitera les chercheurs portugais - qui sont souvent francophones - à contribuer régulièrement à la revue.

\section{Références}

BARRIGA Antónia, 2007, Média, política e opinião. Uma tríade complexa, Lisbonne, ISCTE. BELCHIOR Anna-Maria, 2010, Democracia e representação partidária. A elite parlamentar e os cidadãos, Lisbonne, Imprensa de ciências sociais.

DUARTE Isabel Margarida, 2011, «Política e jornalismo. Algumas práticas discursivas»,

XII Colóquio de Outono. Vozes, discursos e identidades em conflito, A. G. Macedo, C. Sousa, V. Moura éd., Braga, Centro de estudos humanísticos da Universidade do Minho - Edições Húmus, p. 239-248.

EsPírito SANTo Paula, 2006, «A mensagem política na campanha das eleições presidenciais. Análise de conteúdo dos slogans entre 1976 e 2006 », Comunicação \& Cultura, n² 2, Lisbonne, Quimera, Universidade católica portuguesa, p. 83-101.

- 2007, "Comunicação e política nos discursos presidenciais de tomada de posse, 1976-2006 ", Revista de estudos em comunicação, nº 2, p. 185-215, en ligne [http:// www.ec.ubi.pt/ec/02/pdf/Santo-comunicacao-politica-discursos-presidenciaistomada-posse.pdf], consulté le 15 janvier 2013. 
FILIPE Antonio, 2002, As oposições parlamentares em Portugal : práticas e intervenções (1976-2000), Lisbonne, Vega.

FREIRE André, 2006, Esquerda e direita na política europeia. Portugal, Espanha e Grécia em perspectiva comparada, Lisbonne, Imprensa de ciências sociais.

Freire André, Magalhães Pedro, 2002, A abstenção eleitoral em Portugal, Lisbonne, Imprensa de ciências sociais.

Freire André, Lobo Marina Costa, Magalhães Pedro éd., 2007, Eleições e cultura política. Portugal no contexto europeu, Lisbonne, Imprensa de ciências sociais.

GARNIER Roxane, 2001, "La démocratie gouvernante au Portugal», thèse de doctorat, Université Panthéon-Sorbonne.

GRIZE Jean-Blaise, 1990, Logique et langage, Paris-Gap, Ophrys.

Jalalı Carlos, 2007, Partidos e democracia em Portugal, 1974-2005. Da revoluçaõ ao bipartidarismo, Lisbonne, Imprensa de ciências sociais.

LEBLANC Jean-Marc, 2003, "L'ethos, la diachronie, deux facteurs de la variation lexicométrique», Lexicométrica, $\mathrm{n}^{\circ} 4$, en ligne [http://lexicometrica.univ-paris3.fr/ article/numero4.htm], consulté le 15 janvier 2013.

Leblanc Jean-Marc, MARTInez William, 2005, "Positionnements énonciatifs dans les vœux présidentiels sous la Cinquième République. Analyse des marques personnelles par les méthodes de cooccurrence», Corpus, n 4 , décembre, en ligne [http://corpus.revues.org/index347.html], consulté le 15 janvier 2013.

LISI Marco, 2011, Os partidos políticos em Portugal. Continuidade e transformacão, Coimbra, Almedina.

LOPES Fernando Farelo, 2004, Os partidos políticos. Modelos e realidades na Europa Ocidental e em Portugal, Oeiras, Celta Editora.

MARQUES Maria Emilia, 1988, «A complementação verbal. Estudo sociolinguístico», thèse de doctorat ( $4 \mathrm{vol}$.), Lisbonne, Universidade Nova.

MARQUeS Maria Aldina, 2000, «Funcionamento do discurso político parlamentar. A organização enunciativa no debate da interpelação ao governo », thèse de doctorat, Braga, CEHUM - Universidade do Minho.

- 2007, «Discordar no Parlamento. Estratégias de argumentação », Revista galega de filoloxía, n॰8 (Área de filoloxía galega, Departamento de galego-português, francês e lingüística), Universidade da Corunha, p. 99-124.

- 2010a, «The public and private sphere in parliamentary debate. The construction of the addresser in the Portuguese Parliament », European Parliaments under Scrutiny. Discourse Strategies and Interaction Practices, C. Ilie éd., Amsterdam, John Benjamins, p.79-107.

- 2010b, «Circulation et fonction de la médisance en campagne électorale», Cédille, Revista de estudios franceses. Monografias 1, p.65-79, en ligne [http://cedille. webs.ull.es/M1/marques.pdf], consulté le 15 janvier 2013.

- 2012, "Emotions and argumentation in the Portuguese Parliament», Parliamentary Discourses across Cultures. Interdisciplinary Approaches, in L. Ionescu-Ruxandoiu éd., en collab. avec M. Roibu et M.-V. Constantinescu, Newcastle upon Tyne, Cambridge Scholars Publishing, p. 117-132.

Miranda José A. Bragança (de), 1997, Política e modernidade. Linguagem e violência na 
cultura contemporânea, Lisbonne, Colibri.

Pinto Rosalice, 2005, "Le pathos dans les affiches politiques portugaises. Stratégie rhétorico-politique discursive? », Actes du XXVe Colloque d'Albi, Toulouse, Université Toulouse-Le Mirail, p. 319-330.

Ramos Rui Lima, 2011, "Vozes políticas no discurso mediático sobre o ambiente», XII Colóquio de Outono. Vozes, discursos e identidades em conflito, A. G. Macedo, C. Sousa, V. Moura éd., Braga, Centro de estudos humanísticos da Universidade do Minho - Edições Húmus, p. 265-279.

SAlgado Susana, 2010, Os candidatos presidenciais. Construção de imagens e discursos nos media, Coimbra, Minerva Editora.

Sena Nilza Mouzinho (de), 2002, A interpretacão política do debate televisivo, 19741999, Lisbonne, Instituto superior de ciências sociais. 